International Mathematical Forum, Vol. 10, 2015, no. 2, 75 - 93

HIKARI Ltd, www.m-hikari.com

http://dx.doi.org/10.12988/imf.2015.412205

\title{
Some Further Properties for Analytic Functions with Varying Argument Defined by Hadamard Products
}

\author{
Shu-Hai Li, Huo Tang, Li-Na Ma and En Ao \\ School of Mathematics and Statistics \\ Chifeng University \\ Chifeng 024000, Inner Mongolia, China
}

Copyright (c) 2015 Shu-Hai Li, Huo Tang, Li-Na Ma and En Ao. This is an open access article distributed under the Creative Commons Attribution License, which permits unrestricted use, distribution, and reproduction in any medium, provided the original work is properly cited.

\begin{abstract}
The purpose of this paper is to obtain some further properties including coefficients estimates, majorization problems, distortion bounds, extreme points and radius of close-to-convexity, starlikeness and convexity for functions belonging to the class $T U_{\gamma}(\phi, \psi ; \alpha, A, B)$, which are defined by Hadamard products with varying argument.
\end{abstract}

Mathematics Subject Classification: 30C45; 30C50; 26D15

Keywords: analytic functions, Hadamard products, varying argument, subordination, coefficients estimate, majorization 


\section{Introduction}

Let $\mathcal{A}$ denote the class of functions of the form

$$
f(z)=z+\sum_{j=2}^{\infty} a_{j} z^{j},
$$

which are analytic in the open unit disc $\mathbb{U}=\{z \in \mathbb{C}:|z|<1\}$. Let $\mathcal{S}$ be the subclass of $\mathcal{A}$, consisting of analytic and univalent functions. We denote by $\mathcal{S}^{*}(\beta)$ and $\mathcal{K}(\beta)(0 \leq \beta<1)$ the class of starlike of order $\beta$ in $\mathbb{U}$ and the class of convex functions of order $\beta$ in $\mathbb{U}$, respectively. It is well known that $\mathcal{S}^{*}(\beta) \subset \mathcal{S}^{*}(0)=\mathcal{S}^{*}$ and $\mathcal{K}(\beta) \subset \mathcal{K}(0)=\mathcal{K}$.

A function $f(z) \in \mathcal{A}$ is said to be in $U S(\alpha, \beta)$, the class of $\alpha$-uniformly starlike functions of order $\beta(0 \leq \beta<1)$, if $f$ satisfies the condition (see $[1,2]$ )

$$
\Re\left(\frac{z f^{\prime}(z)}{f(z)}\right)>\alpha\left|\frac{z f^{\prime}(z)}{f(z)}-1\right|+\beta, \quad \alpha \geq 0 .
$$

Replacing $f(z)$ in $(1.2)$ by $z f^{\prime}(z)$, we obtain

$$
\Re\left(1+\frac{z f^{\prime \prime}(z)}{f^{\prime}(z)}\right)>\alpha\left|\frac{z f^{\prime \prime}(z)}{f^{\prime}(z)}\right|+\beta, \quad \alpha \geq 0 .
$$

Required for the function $f$ to be in the subclass $U K(\alpha, \beta)$ of $\alpha$-uniformly convex functions of order $\beta$.

Also, by $T_{\gamma}(\gamma \in \mathbb{R})$ we denote the class of functions $f(z) \in \mathcal{A}$ of the form (1.1) for which all of non-vanishing coefficients satisfy the condition

$$
\arg \left(a_{n}\right)=\pi+(1-n) \gamma \quad(n=2,3, \cdots) .
$$

For $\gamma=0$ we obtain the class $T_{0}$ of functions with negative coefficients. Moreover, we define

$$
T=\bigcup_{\gamma \in \mathbb{R}} T_{\gamma}
$$

The class $T$ was introduced by Silverman [3] (see also [4], [5] and [31]). It is called the class of functions with varying argument of coefficients.

For two functions $f$ and $g$, analytic in $\mathbb{U}$, we say that the function $f$ is subordinate to $g$ in $\mathbb{U}$, and write

$$
f(z) \prec g(z) \quad(z \in \mathbb{U}),
$$

if there exists a Schwarz function $\omega$, which (by definition) is analytic in $\mathbb{U}$ with $\omega(0)=0$ and $|\omega(z)|<1(z \in \mathbb{U})$, such that $f(z)=g(\omega(z))(z \in \mathbb{U})$. Furthermore, if the function $g$ is univalent in $\mathbb{U}$, then we have the following equivalence $[6, \mathrm{p} .4]$ :

$$
f(z) \prec g(z)(z \in \mathbb{U}) \Longleftrightarrow f(0)=g(0) \quad \text { and } \quad f(\mathbb{U}) \subset g(\mathbb{U}) \text {. }
$$


Let $f$ and $g$ be analytic in the open unit disk $\mathbb{U}$. We say that $f$ is majorized by $g$ in $\mathbb{U}$ (see [7]) and write

$$
f(z) \ll g(z) \quad(z \in \mathbb{U})
$$

if there exists a function $\varphi(z)$, analytic in $\mathbb{U}$ such that

$$
|\varphi(z)| \leq 1 \quad \text { and } \quad f(z)=\varphi(z) g(z) \quad(z \in \mathbb{U})
$$

It may be noted here that (1.5) is closely related to the concept of quasi-subordination between analytic functions.

For arbitrary fixed real numbers $A$ and $B(-1 \leq B<A \leq 1)$, let $P(A, B)$ denote the class of functions of the form $p(z)=1+\sum_{j=1}^{\infty} p_{j} z^{j}$, which are analytic in $\mathbb{U}$ and satisfies the condition

$$
p(z) \prec \frac{1+A z}{1+B z} \quad(z \in \mathbb{U}) .
$$

The class $P(A, B)$ was introduced and studied by Janowski [8]. We note that a function $f(z) \in P(A, B)$ if and only if

$$
\begin{cases}\left|p(z)-\frac{1-A B}{1-B^{2}}\right|<\frac{A-B}{1-B^{2}}, & (-1<B<A \leq 1 ; z \in \mathbb{U}) ; \\ \Re\{p(z)\}>\frac{1-A}{2}, & (B=-1 ; z \in \mathbb{U}) .\end{cases}
$$

Let

$$
f_{i}(z)=z+\sum_{j=2}^{\infty} a_{j, i} z^{j} \in A,(i=1,2),
$$

then $\left(f_{1} * f_{2}\right)(z)$ be given by

$$
\left(f_{1} * f_{2}\right)(z)=z+\sum_{j=2}^{\infty} a_{j, 1} a_{j, 2} z^{j}
$$

For $\alpha \geq 0,-1 \leq B<A \leq 1$ and for all $z \in \mathbb{U}$, Li et al. [9] defined the subclass $U(\phi, \psi, \alpha, A, B)$ of $\mathcal{A}$ which satisfies the following condition:

$$
\frac{(f * \phi)(z)}{(f * \psi)(z)}-\alpha\left|\frac{(f * \phi)(z)}{(f * \psi)(z)}-1\right| \in P(A, B) \quad(z \in \mathbb{U})
$$

where

$$
\phi(z)=z+\sum_{j=2}^{\infty} \mu_{j} z^{j} \quad \text { and } \quad \psi(z)=z+\sum_{j=2}^{\infty} \eta_{j} z^{j}
$$

are analytic in $\mathbb{U}$ such that $(f * \psi)(z) \neq 0$ and $\mu_{j}>\eta_{j} \geq 0$ for $j \geq 2$. 
For $\alpha=0, A=1-2 \beta(0 \leq \beta<1)$ and $B=-1$, we denote the class $U(\phi, \psi, 0,1-2 \beta,-1)$ by $U(\phi, \psi ; \beta)$. Equivalently, $U(\phi, \psi ; \beta)$ can be expressed in the form

$$
U(\phi, \psi ; \beta)=\left\{f(z) \in \mathcal{A}: \Re\left\{\frac{(f * \phi)(z)}{(f * \psi)(z)}\right\}>\beta, z \in \mathbb{U}\right\} .
$$

Using the results in $[10,11,12]$ and (1.8), we get the following geometric interpretation.

Geometric interpretation. $f(z) \in U(\phi, \psi ; \alpha, A, B)$ if and only if $p(z)=\frac{(f * \phi)(z)}{(f * \psi)(z)}$ takes all values in the conic domain $R_{\alpha}(A, B)$ which is included in the right half plane such that

$$
R_{\alpha}(A, B)=\left\{u+i v: u>\left\{\begin{array}{lc}
\alpha \sqrt{(u-1)^{2}+v^{2}}+\frac{1-A}{1-B}, & -1<B<A \leq 1 ; \\
\alpha \sqrt{(u-1)^{2}+v^{2}}+\frac{1-A}{2}, & B=-1 .
\end{array}\right\}\right.
$$

Denote by $\mathcal{P}(p(\alpha, A, B))$, the family of functions $p$, such that $p \in \mathcal{P}$, where $\mathcal{P}$ denotes the well-known class of Caratheodory functions and $p \prec p(\alpha, A, B)(z)$ in $\mathbb{U}$. The function $p(\alpha, A, B)(z)$ maps the unit disk conformally onto the domain $R_{\alpha}(A, B)$ such that $1 \in R_{\alpha}(A, B)$ and $\partial R_{\alpha}(A, B)$ is a curve defined by the equality

$$
\partial R_{\alpha}(A, B)=\left\{u+i v: u^{2}=\left\{\begin{array}{cc}
\left(\alpha \sqrt{(u-1)^{2}+v^{2}}+\frac{1-A}{1-B}\right)^{2}, & -1<B<A \leq 1 ; \\
\left(\alpha \sqrt{(u-1)^{2}+v^{2}}+\frac{1-A}{2}\right)^{2}, & B=-1 .
\end{array}\right\}\right.
$$

From elementary computations, we see that (1.10) represents conic sections symmetric about the real axis. Thus $R_{\alpha}(A, B)$ is an elliptic domain for $\alpha>1$, a parabolic domain for $\alpha=1$, a hyperbolic domain for $0<\alpha<1$ and the right half plane

$$
u>\left\{\begin{array}{l}
(1-A) /(1-B), \quad-1<B<A \leq 1 \\
(1-A) / 2, \quad B=-1
\end{array}\right.
$$

for $\alpha=0$.

The functions which play the role of extremal functions for these conic regions are given as

$$
p(\alpha, A, B)(z)=\left\{\begin{array}{lc}
\frac{1+\left(1-2 \frac{1-A}{1-B}\right) z}{1-z}, & \alpha=0, \\
1+\frac{2\left(1-\frac{1-A}{1-B}\right)}{\pi^{2}}\left(\log \frac{1+\sqrt{z}}{1-\sqrt{z}}\right)^{2}, & \alpha=1, \\
\frac{1-\frac{1-A}{1-B}}{\alpha^{2}-1} \cos \left\{\left(\frac{2}{\pi} \cos ^{-1} \alpha\right) i \log \frac{1+\sqrt{z}}{1-\sqrt{z}}\right\}-\frac{\alpha^{2}-\frac{1-A}{1-B}}{\alpha^{2}-1}, & 0<\alpha<1, \\
\frac{1-\frac{1-A}{1-B}}{\alpha^{2}-1} \sin \left(\frac{\pi}{2 h(t)}\right) \int_{0}^{\frac{u(z)}{\sqrt{t}}} \frac{1}{\sqrt{1-x^{2}} \sqrt{1-t^{2} x^{2}}} d x+\frac{\alpha^{2}-\frac{1-A}{1-B}}{\alpha^{2}-1}, & \alpha>1,
\end{array}\right.
$$


where $-1<B<A \leq 1, u(z)=\frac{z-\sqrt{t}}{1-\sqrt{t} z}, t \in(0,1)$, every positive number $\alpha$ can be expressed as $\alpha=\cosh \frac{\pi h^{\prime}(t)}{4 h(t)}$, where $h(t)$ is the Legendre's complete elliptic integral of the first kind and $h^{\prime}(t)$ is complementary integral of $h(t)$ (for details, see $[10,11,12]$ ). Also from $(1.11)$, we get the extremum function $p(\alpha, A,-1)(z)(B=-1)$.

Because $p(\alpha, A, B)(z)$ is a convex univalent function, so we can write the class $U(\phi, \psi ; \alpha, A, B)$ in the subordination form

$$
f(z) \in U(\phi, \psi ; \alpha, A, B) \Longleftrightarrow f(z) \in \mathcal{A} \quad \text { and } \quad p(z)=\frac{(f * \phi)(z)}{(f * \psi)(z)} \prec p(\alpha, A, B)(z) \quad(z \in \mathbb{U}) .
$$

Let us denote

$$
\begin{gathered}
T U_{\gamma}(\phi, \psi ; \alpha, A, B)=T_{\gamma} \cap U(\phi, \psi ; \alpha, A, B), \\
T U S_{\gamma}(\alpha, \beta)=T_{\gamma} \cap U S(\alpha, \beta), \quad T U K_{\gamma}(\alpha, \beta)=T_{\gamma} \cap U K(\alpha, \beta) .
\end{gathered}
$$

For suitable choices of $\phi, \psi$ and by specializing the parameters $\alpha, A, B$ involved in the class $U(\phi, \psi ; \alpha, A, B)$, we also obtain the following subclasses which were studied in many earlier works:

(i) $U\left(\frac{z}{(1-z)^{2}}, \frac{z}{1-z} ; \alpha, 1-2 \beta,-1\right)=U S(\alpha, \beta)$ and $U\left(\frac{z+z^{2}}{(1-z)^{3}}, \frac{z}{(1-z)^{2}} ; \alpha, 1-2 \beta,-1\right)=U K(\alpha, \beta)$ (Shams et al. [1] and Shams and Kulkarni [2]).

(ii) $U\left(\frac{z}{(1-z)^{2}}, \frac{z}{1-z} ; 0, A, B\right)=S^{*}(A, B)$ and $U\left(\frac{z+z^{2}}{(1-z)^{3}}, \frac{z}{(1-z)^{2}} ; 0, A, B\right)=K(A, B)$ (Janowski [8] and Padmanabhan et al. [13]). For example, we have $S^{*}(1,-1)=S^{*}$ and $K(1,-1)=K$.

(iii) $U\left(\frac{z}{(1-z)^{2}}, \frac{z}{1-z} ; \alpha, 1,-1\right)=U S(\alpha)$ and $U\left(\frac{z+z^{2}}{(1-z)^{3}}, \frac{z}{(1-z)^{2}} ; \alpha, 1,-1\right)=U K(\alpha)$ (Goodman[14], Ma and Minda [15] and Rønning [16]).

(iv) $U\left(z+\sum_{j=2}^{\infty} j^{n+1} z^{j}, z+\sum_{j=2}^{\infty} j^{n} z^{j} ; \alpha, 1-2 \beta,-1\right)=U S_{n}(\alpha, \beta)$ (Bharati et al. [17] and Kuang et al. [18]).

(v) $U\left(z+\sum_{j=2}^{\infty} j^{m} z^{j}, z+\sum_{j=2}^{\infty} j^{n} z^{j} ; \alpha, 1-2 \beta,-1\right)=U S_{m, n}(\alpha, \beta)(0 \leq \alpha, 0 \leq \beta<1)$ (Eker and Owa [19] and Srivastava and Eker [20]).

(vi) $U\left(z+\sum_{j=2}^{\infty} j^{m} \lambda_{j} z^{j}, z+\sum_{j=2}^{\infty} j^{n} \tau_{j} z^{j} ; \alpha,(1-\sigma a)+\sigma b, b\right)=E_{m, n}(\phi, \psi ; a, b, \sigma, \alpha)\left(\lambda_{j}>\tau_{j} \geq\right.$ $0 ;-1 \leq b<a \leq 1,0 \leq \sigma<1$ ) (Srivastava et al. [21]). 
(vii) $U\left(z+\sum_{j=2}^{\infty} j \phi_{j}^{\tau}\left(\alpha_{1}, \lambda, l, m\right) z^{j}, z+\sum_{j=2}^{\infty} \phi_{j}^{\tau}\left(\alpha_{1}, \lambda, l, m\right) z^{j} ; \alpha, \beta,-1\right)=U S_{m}^{l}(\tau, \lambda, \alpha, \beta)(\alpha \geq$ $0,0 \leq \beta<1)$ (Srivastava et al. [22])

(viii) $U\left(z+\sum_{j=2}^{\infty} j\left(\frac{1+b}{j+b}\right)^{s} z^{j}, z+\sum_{j=2}^{\infty}\left(\frac{1+b}{j+b}\right)^{s} z^{j} ; \alpha, 1-\beta,-1\right)=U S T_{s, b}(\sigma, \beta)$

$(s \in \mathbb{C} ; b \in \mathbb{C} \backslash\{0,-1,-2, \cdots\} ; 0 \leq \beta<1$ ) (Sun et al. [23]).

(ix) $U\left(z+\sum_{j=2}^{\infty} j^{m} z^{j}, z+\sum_{j=2}^{\infty} j^{n} z^{j} ; \alpha, A, B\right)=U S_{m, n}(\alpha, A, B)(0 \leq \alpha, 0 \leq \beta<1)(\mathrm{Li}$ and Tang [24], Aouf et al. [25] and El-Ashwah et al. [26]).

In this paper, we aim to obtain some further properties, such as coefficients estimates, majorization problems, distortion bounds, extreme points and radius of close-to-convexity, starlikeness and convexity for functions belonging to the class $T U_{\gamma}(\phi, \psi ; \alpha, A, B)$.

\section{Preliminary results}

We need the following results in our next investigation.

Lemma 2.1. Let $f(z) \in U(\phi, \psi ; \alpha, A, B)$. Then

$f(z) \in U(\phi, \psi ; \alpha, A, B) \Longrightarrow\left\{\begin{array}{l}f(z) \in U\left(\phi, \psi ; \frac{(1-A)-\alpha(1-B)}{(1-\alpha)(1-B)}\right), \quad-1<B<A \leq 1, \alpha(1-B) \leq 1-A ; \\ f(z) \in U\left(\phi, \psi ; \frac{(1-A)-2 \alpha}{2(1-\alpha)}\right), \quad B=-1,2 \alpha \leq 1-A .\end{array}\right.$

Proof. Let $f(z) \in U(\phi, \psi ; \alpha, A, B)$. Then we obtain

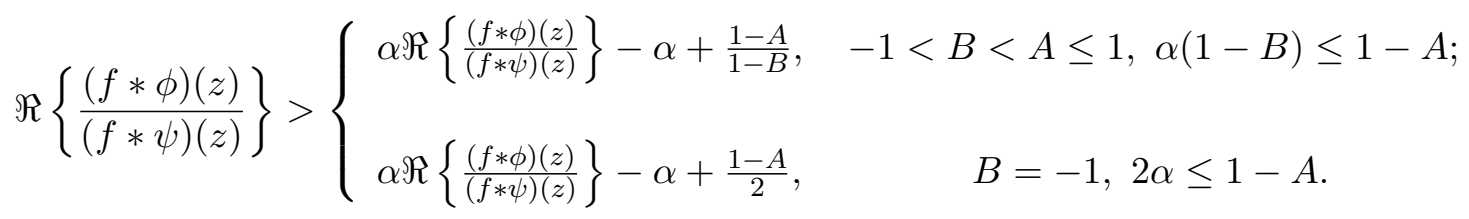

or, equivalently,

$$
\Re\left\{\frac{(f * \phi)(z)}{(f * \psi)(z)}\right\}>\left\{\begin{array}{lc}
\frac{(1-A)-\alpha(1-B)}{(1-\alpha)(1-B)}, & -1<B<A \leq 1, \alpha(1-B) \leq 1-A ; \\
\frac{(1-A)-2 \alpha}{2(1-\alpha)}, & B=-1,2 \alpha \leq 1-A .
\end{array}\right.
$$

If $-1<B<A \leq 1$ and $\alpha(1-B) \leq 1-A$, then we have

$$
0 \leq \frac{(1-A)-\alpha(1-B)}{(1-\alpha)(1-B)}<1
$$


Also if $B=-1$ and $2 \alpha \leq 1-A$, then we obtain

$$
0 \leq \frac{(1-A)-2 \alpha}{2(1-\alpha)}<1
$$

Thus we prove Lemma 2.1.

Lemma $2.2([9])$. Let $f(z)$ be the function of the form (1.1). Then $f(z) \in T U_{\gamma}(\phi, \psi ; \alpha, A, B)$ if and only if

$$
\sum_{j=2}^{\infty} \phi_{j}\left(\mu_{j}, \eta_{j}, \alpha, A, B\right)\left|a_{j}\right| \leq A-B
$$

where

$$
\begin{gathered}
\phi_{j}\left(\mu_{j}, \eta_{j}, \alpha, A, B\right)=(1+(1+|B| \alpha))\left(\mu_{j}-\eta_{j}\right)+\left|B \mu_{j}-A \eta_{j}\right| \\
\left(\alpha \geq 0,-1 \leq B<A \leq 1, \mu_{j}>\eta_{j} \geq 0, j \geq 2\right) .
\end{gathered}
$$

Lemma 2.3 ([27, p.3]). Let $\alpha \geq 0$ and $-1 \leq B<A \leq 1$. If $\omega(z)$ is an analytic function with $\omega(0)=1$, then we have

$$
\omega-\alpha|\omega-1| \prec \frac{1+A z}{1+B z} \Longleftrightarrow \omega\left(1-\alpha e^{-i \phi}\right)+\alpha e^{-i \phi} \prec \frac{1+A z}{1+B z} \quad(\phi \in \mathbb{R}) .
$$

Lemma 2.4 ([32]). Let $\varphi(z)$ be analytic in $\mathbb{U}$ satisfy $|\varphi(z)| \leq 1$ for $z \in \mathbb{U}$. Then

$$
\left|\varphi^{\prime}(z)\right| \leq \frac{1-|\varphi(z)|^{2}}{1-|z|^{2}} \quad(z \in \mathbb{U})
$$

Lemma 2.5. Let $f(z) \in U(\phi, \psi ; \alpha, A, B)$. Then

$$
f(z) \in U(\phi, \psi ; \alpha, A, B) \Longrightarrow \begin{cases}f(z) \in U\left(\phi, \psi ; \frac{1-A}{1-B}\right), & -1<B<A \leq 1 \\ f(z) \in U\left(\phi, \psi ; \frac{1-A}{2}\right), & B=-1 .\end{cases}
$$

Proof. By virtue of (1.8), (1.10) and the properties of the domain $R_{\alpha}(A, B)$, we obtain

$$
\Re\left\{\frac{(f * \phi)(z)}{(f * \psi)(z)}\right\}>\left\{\begin{array}{lc}
\frac{(1-B) \alpha+1-A}{(\alpha+1)(1-B)}, & -1<B<A \leq 1 ; \\
\frac{2 \alpha+1-A}{2(\alpha+1)}, & B=-1 .
\end{array}\right.
$$

Thus we prove Lemma 2.5 . 
Lemma 2.6. If $f(z) \in T U_{\gamma}(\phi, \psi ; \alpha, A, B)$ and

$$
\phi_{j}\left(\mu_{j}, \eta_{j}, \alpha, A, B\right) \geq \phi_{p+1}\left(\mu_{p+1}, \eta_{p+1}, \alpha, A, B\right)(j \geq p+1, p \geq 2),
$$

then

$$
\sum_{j=p+1}^{\infty} a_{j} \leq \frac{(A-B)-\sum_{j=2}^{p} \phi_{j}\left(\mu_{j}, \eta_{j}, \alpha, A, B\right) a_{j}}{\phi_{p+1}\left(\mu_{p+1}, \eta_{p+1}, \alpha, A, B\right)}=A_{j} .
$$

Proof. In view of Lemma 2.2, we can write that

$$
\sum_{j=p+1}^{\infty} \phi_{p+1}\left(\mu_{p+1}, \eta_{p+1}, \alpha, A, B\right) a_{j} \leq(A-B)-\sum_{j=2}^{p} \phi_{j}\left(\mu_{j}, \eta_{j}, \alpha, A, B\right) a_{j} .
$$

Then from (2.7) and (2.9), we have

$$
\phi_{p+1}\left(\mu_{p+1}, \eta_{p+1}, \alpha, A, B\right) \sum_{j=p+1}^{\infty} a_{j} \leq(A-B)-\sum_{j=2}^{p} \phi_{j}\left(\mu_{j}, \eta_{j}, \alpha, A, B\right) a_{j} .
$$

Thus we obtain

$$
\sum_{j=p+1}^{\infty} a_{j} \leq \frac{(A-B)-\sum_{j=2}^{p} \phi_{j}\left(\mu_{j}, \eta_{j}, \alpha, A, B\right) a_{j}}{\phi_{p+1}\left(\mu_{p+1}, \eta_{p+1}, \alpha, A, B\right)}
$$

Also, we easily obtain

Lemma 2.7. If $f(z) \in T U_{\gamma}(\phi, \psi ; \alpha, A, B)$ and $\phi_{j}\left(\mu_{j}, \eta_{j}, \alpha, A, B\right)$ defined by (2.3) satisfies (2.7), then

$$
\sum_{j=p+1}^{\infty} j a_{j} \leq \frac{(A-B)-\sum_{j=2}^{p} \phi_{j}\left(\mu_{j}, \eta_{j}, \alpha, A, B\right) a_{j}}{\frac{1}{p+1} \phi_{p+1}\left(\mu_{p+1}, \eta_{p+1}, \alpha, A, B\right)}=B_{j}
$$

\section{Main Results}

Theorem 3.1. Let $f(z) \in U(\phi, \psi ; \alpha, A, B)$. Then

$$
\left|a_{2}\right| \leq\left\{\begin{array}{l}
\frac{2(1-(1-A) /(1-B))}{\left(\mu_{2}-\eta_{2}\right)(1-\alpha)}, \quad-1<B<A \leq 1, \quad \alpha(1-B) \leq 1-A \\
\frac{2(1-(1-A) / 2)}{\left(\mu_{2}-\eta_{2}\right)(1-\alpha)}, \quad B=-1,2 \alpha \leq 1-A .
\end{array}\right.
$$

and

$$
\left|a_{j}\right| \leq\left\{\begin{array}{l}
\frac{2(1-(1-A) /(1-B))}{\left(\mu_{j}-\eta_{j}\right)(1-\alpha)} \prod_{k=1}^{j-2}\left(1+\frac{2(1-(1-A) /(1-B))}{\left(\mu_{k+1}-\eta_{k+1}\right)(1-\alpha)}\right), \quad-1<B<A \leq 1, \alpha(1-B) \leq 1-A ; j \geq 3 \\
\frac{2(1-(1-A) / 2)}{\left(\mu_{j}-\eta_{j}\right)(1-\alpha)} \prod_{k=1}^{j-2}\left(1+\frac{2(1-(1-A) / 2)}{\left(\mu_{k+1}-\eta_{k+1}\right)(1-\alpha)}\right), \quad B=-1, \quad 2 \alpha \leq 1-A ; j \geq 3 .
\end{array}\right.
$$


Proof. Suppose that $f \in U(\phi, \psi ; \alpha, A, B)$. Then, by Lemma 2.1, we obtain

$$
\Re\left\{\frac{(f * \phi)(z)}{(f * \psi)(z)}\right\}>\left\{\begin{array}{lc}
\frac{(1-A)-\alpha(1-B)}{(1-\alpha)(1-B)}, & -1<B<A \leq 1, \alpha(1-B) \leq 1-A ; \\
\frac{(1-A)-2 \alpha}{2(1-\alpha)}, & B=-1,2 \alpha \leq 1-A .
\end{array}\right.
$$

Let us define the function $p(z)$ by

$$
p(z)= \begin{cases}\frac{(1-\alpha) \frac{(f * \phi)(z)}{(f * \psi)(z)}-\frac{(1-A)-\alpha(1-B)}{(1-B)}}{\frac{A-B}{1-B}}, & -1<B<A \leq 1, \alpha(1-B) \leq 1-A ; \\ \frac{(1-\alpha) \frac{(f * \phi)(z)}{(f * \psi)(z)}-\frac{(1-A)-2 \alpha}{2}}{\frac{1+A}{2}}, & B=-1,2 \alpha \leq 1-A .\end{cases}
$$

Hence $p(z)$ is analytic in $\mathrm{U}$ with $p(0)=1$ and $\Re p(z)>0(z \in U)$. Let

$$
p(z)=1+c_{1} z+c_{2} z^{2}+\ldots
$$

So we get

$$
\frac{(f * \phi)(z)}{(f * \psi)(z)}=\left\{\begin{array}{l}
1+\frac{A-B}{(1-\alpha)(1-B)}\left(c_{1} z+c_{2} z^{2}+\ldots\right), \quad-1<B<A \leq 1, \alpha(1-B) \leq 1-A \\
1+\frac{1+A}{2(1-\alpha)}\left(c_{1} z+c_{2} z^{2}+\ldots\right), \quad B=-1,2 \alpha \leq 1-A .
\end{array}\right.
$$

or, equivalently,

$$
(f * \phi)(z)-(f * \psi)(z)=\left\{\begin{array}{l}
\frac{A-B}{(1-\alpha)(1-B)}((f * \psi)(z))\left(c_{1} z+c_{2} z^{2}+\ldots\right), \quad-1<B<A \leq 1, \alpha(1-B) \leq 1-A \\
\frac{1+A}{2(1-\alpha)}((f * \psi)(z))\left(c_{1} z+c_{2} z^{2}+\ldots\right), \quad B=-1,2 \alpha \leq 1-A
\end{array}\right.
$$

which implies that

$\left(\mu_{j}-\eta_{j}\right) a_{j}=\left\{\begin{array}{l}\frac{A-B}{(1-\alpha)(1-B)}\left(c_{j-1}+a_{2} c_{j-2}+\ldots+a_{j-1} c_{1}\right), \quad-1<B<A \leq 1, \alpha(1-B) \leq 1-A \\ \frac{1+A}{2(1-\alpha)}\left(c_{j-1}+a_{2} c_{j-2}+\ldots+a_{j-1} c_{1}\right), \quad B=-1,2 \alpha \leq 1-A .\end{array}\right.$

Applying the coefficient estimates $\left|c_{j}\right| \leq 2(j \geq 1)$ (see [33]), we obtain

$$
\left|a_{j}\right| \leq\left\{\begin{array}{l}
\frac{2(A-B)}{\left(\mu_{j}-\eta_{j}\right)(1-\alpha)(1-B)}\left(1+a_{2}+\ldots+a_{j-1}\right) \quad-1<B<A \leq 1, \alpha(1-B) \leq 1-A ; \\
\frac{(1+A)}{\left(\mu_{j}-\eta_{j}\right)(1-\alpha)}\left(1+a_{2}+\ldots+a_{j-1}\right), \quad B=-1,2 \alpha \leq 1-A .
\end{array}\right.
$$


For $j=2$,

$$
\left|a_{2}\right| \leq\left\{\begin{array}{l}
\frac{2(A-B)}{\left(\mu_{2}-\eta_{2}\right)(1-\alpha)(1-B)}, \quad-1<B<A \leq 1, \quad \alpha(1-B) \leq 1-A \\
\frac{1+A}{\left(\mu_{2}-\eta_{2}\right)(1-\alpha)}, \quad B=-1,2 \alpha \leq 1-A .
\end{array}\right.
$$

which proves (3.1).

For $j=3$,

$$
\left|a_{3}\right| \leq\left\{\begin{array}{l}
\frac{2(A-B)}{\left(\mu_{3}-\eta_{3}\right)(1-\alpha)(1-B)}\left(1+\frac{2(A-B)}{\left(\mu_{2}-\eta_{2}\right)(1-\alpha)(1-B)}\right), \quad-1<B<A \leq 1, \alpha(1-B) \leq 1-A \\
\frac{1+A}{\left(\mu_{2}-\eta_{2}\right)(1-\alpha)}\left(1+\frac{1+A}{\left(\mu_{2}-\eta_{2}\right)(1-\alpha)}\right), \quad B=-1,2 \alpha \leq 1-A .
\end{array}\right.
$$

Therefore (3.2) holds true for $j=3$. Assume that (3.3) is true for $j=m$.

If $-1<B<A \leq 1$ and $\alpha(1-B) \leq 1-A$, then we have

$$
\begin{gathered}
\left|a_{m+1}\right| \leq \frac{2(A-B)}{\left(\mu_{m+1}-\eta_{m+1}\right)(1-\alpha)(1-B)}\left\{1+\frac{2(A-B)}{\left(\mu_{2}-\eta_{2}\right)(1-\alpha)(1-B)}\right. \\
+\frac{2(A-B)}{\left(\mu_{3}-\eta_{3}\right)(1-\alpha)(1-B)}\left(1+\frac{2(A-B)}{\left(\mu_{2}-\eta_{2}\right)(1-\alpha)(1-B)}\right)+\ldots \\
\left.+\frac{2(A-B)}{\left(\mu_{m-1}-\eta_{m-1}\right)(1-\alpha)(1-B)} \prod_{k=1}^{m-2}\left(1+\frac{2(A-B)}{\left(\mu_{k+1}-\eta_{k+1}\right)(1-\alpha)(1-B)}\right)\right\} . \\
=\frac{2(A-B)}{\left(\mu_{m+1}-\eta_{m+1}\right)(1-\alpha)(1-B)} \prod_{k=1}^{m-1}\left(1+\frac{2(A-B)}{\left(\mu_{k+1}-\eta_{k+1}\right)(1-\alpha)(1-B)}\right)
\end{gathered}
$$

Also, if $B=-1$ and $2 \alpha \leq 1-A$, then we have

$$
\left|a_{m+1}\right| \leq \frac{1+A}{\left(\mu_{m+1}-\eta_{m+1}\right)(1-\alpha)} \prod_{k=1}^{m-1}\left(1+\frac{1+A}{\left(\mu_{k+1}-\eta_{k+1}\right)(1-\alpha)}\right) .
$$

So (3.2) is true for $j=m+1$. Consequently, using the mathematical induction, we get that (3.2) holds true for all $j \geq 3$.

Remark 3.1. Taking $\alpha=0, \mu_{j}=j, \eta_{j}=1, B=-1$ and $A=1-2 \beta(0 \leq \beta<1)$ in Theorem 3.1, we obtain the results of Robertson [34].

Theorem 3.2. Let the function $f \in \mathcal{A}$ and suppose that $f \in T U_{\gamma}(\phi, \psi ; \alpha, A, B)(0 \leq \alpha \neq 1)$. If $(f * \phi)(z)$ is majorized by $(f * \psi)(z)$ and $|(f * \phi)(z)| \leq\left|z(f * \psi)^{\prime}(z)\right|$, then

$$
\left|(f * \phi)^{\prime}(z)\right| \leq\left|(f * \psi)^{\prime}(z)\right| \quad\left(|z| \leq r_{0}\right),
$$


where $r_{0}=r_{0}(\alpha, A, B)$ is the smallest positive root of the equation

$$
\begin{gathered}
{\left[\frac{A-B}{|1-\alpha|}+|B|\right] r^{3}-[1+2|B|] r^{2}-\left[\frac{A-B}{|1-\alpha|}+|B|+2\right] r+1=0} \\
\left(z \in \mathbb{U} ;-1 \leq B<A \leq 1 ; 0 \leq \delta \leq r_{0} ;\left[\frac{A-B}{|1-\alpha|}+|B|\right] \delta \leq 1\right) .
\end{gathered}
$$

Proof. Suppose that $f \in T U_{\gamma}(\phi, \psi ; \alpha, A, B)$. Then, by Lemma 2.3, we obtain

$$
\frac{f(z) * \phi(z)}{f(z) * \psi(z)}\left(1-\alpha e^{-i \phi}\right)+\alpha e^{-i \phi} \prec \frac{1+A z}{1+B z}
$$

or, equivalently,

$$
\frac{f(z) * \phi(z)}{f(z) * \psi(z)} \prec \frac{1+\left(\frac{A-\alpha B e^{-i \phi}}{1-\alpha e^{-i \phi}}\right) z}{1+B z}
$$

which holds true for all $z \in \mathbb{U}$.

We find from (3.6) that

$$
\frac{f(z) * \phi(z)}{f(z) * \psi(z)}=\frac{1+\left(\frac{A-\alpha B e^{-i \phi}}{1-\alpha e^{-i \phi}}\right) \omega(z)}{1+B \omega(z)}
$$

where $\omega(z)=c_{1} z+c_{2} z^{2}+\cdots \in \mathcal{W}, \mathcal{W}$ denotes the well known class of the bounded analytic functions in $\mathbb{U}$ and satisfies the conditions:

$$
\omega(0)=0, \quad|\omega(z)| \leq|z| \quad(z \in \mathbb{U})
$$

From (3.7), we get

$$
|(f * \psi)(z)| \leq \frac{1+|B||z|}{1-\left(\frac{A-B}{|1-\alpha|}+|B|\right)|z|}|(f * \phi)(z)| .
$$

Next, since $(f * \phi)(z)$ is majorized by $(f * \psi)(z)$ in $\mathbb{U}$, from (1.6), we have

$$
(f * \phi)(z)=\varphi(z)(f * \psi)(z) .
$$

Differentiating it with respect to $z$ and multiplying by $z$, we get

$$
(f * \phi)^{\prime}(z)=\varphi^{\prime}(z)(f * \psi)(z)+\varphi(z)(f * \psi)^{\prime}(z) .
$$

Thus, by Lemma 2.4, (3.8) and (3.9), we get

$$
\left|(f * \phi)^{\prime}(z)\right| \leq\left[|\varphi(z)|+\frac{1-|\varphi(z)|^{2}}{1-|z|^{2}} \cdot \frac{(1+|B||z|)|z|}{\left[1-\left(\frac{A-B}{|1-\alpha|}+|B|\right)|z|\right]}\right]\left|(f * \psi)^{\prime}(z)\right|,
$$

which upon setting

$$
|z|=r \quad \text { and } \quad|\varphi(z)|=\rho \quad(0 \leq \rho \leq 1)
$$


leads us to the inequality

$$
\left|(f * \phi)^{\prime}(z)\right| \leq\left[\frac{\psi(\rho)}{\left(1-r^{2}\right)\left[1-\left(\frac{A-B}{|1-\alpha|}+|B|\right) r\right]}\right]\left|(f * \psi)^{\prime}(z)\right|,
$$

where

$$
\psi(\rho)=-r(1+|B| r) \rho^{2}+\left(1-r^{2}\right)\left[1-\left(\frac{A-B}{|1-\alpha|}+|B|\right) r\right] \rho+r(1+|B| r)
$$

takes its maximum value at $\rho=1$, with $r_{0}=r_{0}(\alpha, A, B)$, where $r_{0}=r_{0}(\alpha, A, B)$ is the smallest positive root of (3.5). Furthermore, if $0 \leq \delta \leq r_{0}(\alpha, A, B)$, then the function $\psi(\rho)$ defined by

$$
\psi(\rho)=-\delta(1+|B| \delta) \rho^{2}+\left(1-\delta^{2}\right)\left[1-\left(\frac{A-B}{|1-\alpha|}+|B|\right) \delta\right] \rho+(1+|B| \delta) \delta
$$

is an increasing function on the interval $0 \leq \rho \leq 1$, so that

$$
\psi(\rho) \leq \psi(1)=\left(1-\delta^{2}\right)\left[1-\left(\frac{A-B}{|1-\alpha|}+|B|\right) \delta\right] \quad\left(0 \leq \rho \leq 1 ; 0 \leq \delta \leq r_{0}(\alpha, A, B)\right)
$$

Hence, upon setting $\rho=1$ in (3.13), we conclude that (3.4) of Theorem 3.2 holds true for $|z| \leq r_{0}=r_{0}(\alpha, A, B)$, which completes the proof of Theorem 3.2.

Theorem 3.3. Let the function $f \in \mathcal{A}$ and suppose that $f \in T U_{\gamma}(\phi, \psi ; 1, A, B)(\alpha=1)$. If $(f * \phi)(z)$ is majorized by $(f * \psi)(z)$ and $|(f * \phi)(z)| \leq\left|z(f * \psi)^{\prime}(z)\right|$, then

$$
\left|(f * \phi)^{\prime}(z)\right| \leq\left|(f * \psi)^{\prime}(z)\right|
$$

where $r_{0}=r_{0}(A, B)$ is the smallest positive root of the equation

$$
\chi(A, B)=\left\{\begin{array}{l}
\left(\frac{1-A}{1-B}\right) r^{2}-\left(3+\frac{1-A}{1-B}\right) r+1=0, \quad-1<B<A \leq 1 ; \\
\left(\frac{1-A}{2}\right) r^{2}-\left(3+\frac{1-A}{2}\right) r+1=0, \quad B=-1 . \\
\left(z \in \mathbb{U} ;-1 \leq B<A \leq 1 ; r_{0} \geq 0\right) .
\end{array}\right.
$$

Proof. Suppose that $f \in T U_{\gamma}(\phi, \psi ; 1, A, B)$. Then, by Lemma 2.5, we obtain

$$
\Re\left\{\frac{(f * \phi)(z)}{(f * \psi)(z)}\right\}> \begin{cases}\frac{(1-B)+1-A}{2(1-B)}, & -1<B<A \leq 1 ; \\ \frac{3-A}{4}, & B=-1,\end{cases}
$$

or, equivalently,

$$
\frac{(f * \phi)(z)}{(f * \psi)(z)} \prec \begin{cases}\frac{1+\left(1-\frac{(1-B)+1-A}{(1-B)}\right) z}{1-z}, & -1<B<A \leq 1 ; \\ \frac{1+\left(1-\frac{3-A}{2}\right) z}{1-z}, & B=-1,\end{cases}
$$


which holds true for all $z \in \mathbb{U}$.

We find from (3.17) that

$$
\frac{(f * \phi)(z)}{(f * \psi)(z)}=\left\{\begin{array}{lc}
\frac{1+\left(1-\frac{(1-B)+1-A}{(1-B)}\right) \omega(z)}{1-\omega(z)}, & -1<B<A \leq 1 ; \\
\frac{1+\left(1-\frac{3-A}{2}\right) \omega(z)}{1-\omega(z)}, & B=-1,
\end{array}\right.
$$

where $\omega(z)=c_{1} z+c_{2} z^{2}+\cdots \in \mathcal{W}$. The remainder of Theorem 3.3 is analogous to the proof of Theorem 3.2, so we omit the details involved.

Theorem 3.4. If $f(z) \in T U_{\gamma}(\phi, \psi ; \alpha, A, B)$ and $\phi_{j}\left(\mu_{j}, \eta_{j}, \alpha, A, B\right)$ defined by (2.3) satisfies (2.7), then for $|z|=r<1$

$$
r-r^{2} \sum_{j=2}^{p}\left|a_{j}\right|-\left|A_{j}\right| r^{p+1} \leq|f(z)| \leq r+r^{2} \sum_{j=2}^{p}\left|a_{j}\right|+\left|A_{j}\right| r^{p+1}
$$

and

$$
1-r \sum_{j=2}^{p}\left|a_{j}\right|-\left|B_{j}\right| r^{p} \leq\left|f^{\prime}(z)\right| \leq 1+r \sum_{j=2}^{p}\left|a_{j}\right|+\left|B_{j}\right| r^{p} .
$$

where $A_{j}$ and $B_{j}$ are given by (2.8) and (2.10), respectively.

Proof. Let $f(z)$ be given by (1.1). For $|z|=r<1$, by using Lemma 2.6, we have

$$
\begin{aligned}
|f(z)| & \leq|z|+\sum_{j=2}^{p}\left|a_{j}\right||z|^{j}+\sum_{j=p+1}^{\infty}\left|a_{j}\right||z|^{j} \\
& \leq|z|+|z|^{2} \sum_{j=2}^{p}\left|a_{j}\right|+|z|^{p+1} \sum_{j=p+1}^{\infty}\left|a_{j}\right| \\
& \leq r+r^{2} \sum_{j=2}^{p}\left|a_{j}\right|+\left|A_{j}\right| r^{p+1}
\end{aligned}
$$

and

$$
\begin{aligned}
|f(z)| & \geq|z|-\sum_{j=2}^{p}\left|a_{j}\right||z|^{j}-\sum_{j=p+1}^{\infty}\left|a_{j}\right||z|^{j} \\
& \geq|z|-|z|^{2} \sum_{j=2}^{p}\left|a_{j}\right|-|z|^{p+1} \sum_{j=p+1}^{\infty}\left|a_{j}\right| \\
& \geq r-r^{2} \sum_{j=2}^{p}\left|a_{j}\right|-\left|A_{j}\right| r^{p+1}
\end{aligned}
$$

Furthermore for $|z|=r<1$, by using Lemma 2.7, we obtain

$$
\begin{aligned}
\left|f^{\prime}(z)\right| & \leq 1+\sum_{j=2}^{p} j\left|a_{j}\right||z|^{j-1}+\sum_{j=p+1}^{\infty} j\left|a_{j}\right||z|^{j-1} \\
& \leq 1+|z| \sum_{j=2}^{p} j\left|a_{j}\right|+|z|^{p} \sum_{j=p+1}^{\infty} j\left|a_{j}\right| \\
& \leq 1+r \sum_{j=2}^{p}\left|a_{j}\right|+\left|B_{j}\right| r^{p}
\end{aligned}
$$

and 


$$
\begin{aligned}
\left|f^{\prime}(z)\right| & \geq 1-\sum_{j=2}^{p} j\left|a_{j}\right||z|^{j-1}-\sum_{j=p+1}^{\infty} j\left|a_{j}\right||z|^{j-1} \\
& \geq 1-|z| \sum_{j=2}^{p} j\left|a_{j}\right|-|z|^{p} \sum_{j=p+1}^{\infty} j\left|a_{j}\right| \\
& \geq 1-r \sum_{j=2}^{p}\left|a_{j}\right|-\left|B_{j}\right| r^{p}
\end{aligned}
$$

thus we have (3.19).

Theorem 3.5. Let the function $f(z)$ defined by (1.1) satisfy (1.4). We define

$$
f_{1}(z)=z, \quad f_{j}(z)=z-\frac{A-B}{\phi_{j}\left(\mu_{j}, \eta_{j}, \alpha, A, B\right)} e^{i(1-j) \gamma} z^{j}(j=2,3, \cdots),
$$

where $\phi_{j}\left(\mu_{j}, \eta_{j}, \alpha, A, B\right)$ is given by (2.3). Then $f(z) \in T U_{\gamma}(\phi, \psi ; \alpha, A, B)$ if and only if it can be expressed in the form

$$
f(z)=\sum_{j=1}^{\infty} \lambda_{j} f_{j}(z)
$$

where $\lambda_{j}>0$ and $\sum_{j=1}^{\infty} \lambda_{j}=1$.

Proof. Suppose that

$$
f(z)=\sum_{j=1}^{\infty} \lambda_{j} f_{j}(z)=z-\sum_{j=1}^{\infty} \lambda_{j} \frac{A-B}{\phi_{j}\left(\mu_{j}, \eta_{j}, \alpha, A, B\right)} e^{i(1-j) \gamma} z^{j}
$$

Then

$$
\begin{aligned}
\sum_{j=1}^{\infty} \phi_{j}\left(\mu_{j},\right. & \left.\eta_{j}, \alpha, A, B\right)\left|\lambda_{j} \frac{A-B}{\phi_{j}\left(\mu_{j}, \eta_{j}, \alpha, A, B\right)} e^{i(1-j) \gamma}\right|=(A-B) \sum_{j=2}^{\infty} \lambda_{j} \\
& =(A-B)\left(1-\lambda_{1}\right) \\
& <A-B .
\end{aligned}
$$

By Lemma 2.2, we have $f(z) \in T U_{\gamma}(\phi, \psi ; \alpha, A, B)$.

Conversely, suppose that $f(z) \in T U_{\gamma}(\phi, \psi ; \alpha, A, B)$. Since

$$
\left|a_{j}\right| \leq \frac{A-B}{\phi_{j}\left(\mu_{j}, \eta_{j}, \alpha, A, B\right)}(j=2,3, \cdots),
$$

we may set

$$
\lambda_{j}=\frac{\phi_{j}\left(\mu_{j}, \eta_{j}, \alpha, A, B\right)}{(A-B)\left|e^{i(1-j) \gamma \mid}\right|}\left|a_{j}\right|(j=2,3, \cdots)
$$

and

$$
\lambda_{1}=1-\sum_{j=2}^{\infty} \lambda_{j} .
$$

Then

$$
f(z)=\sum_{j=1}^{\infty} \lambda_{j} f_{j}(z) .
$$


This completes the proof of Theorem 3.5.

Theorem 3.6. Let the function $f(z)$ defined by (1.1) be in the class $T U(\phi, \psi ; \alpha, A, B)$, and $\phi_{j}\left(\mu_{j}, \eta_{j}, \alpha, A, B\right)$ be given by (2.3). Then, we have

(1) The function $f(z)$ is close-to-convex of $\mu(0 \leq \mu<1)$ in $|z|<r_{1}$ where

$$
r_{1}=\inf _{j}\left\{\frac{(1-\mu) \phi_{j}\left(\mu_{j}, \eta_{j}, \alpha, A, B\right)}{j(A-B)}\right\}^{\frac{1}{j-1}}(j \geq 2)
$$

(2) The function $f(z)$ is starlike of $\eta(0 \leq \eta<1)$ in $|z|<r_{2}$ where

$$
r_{2}=\inf _{j}\left\{\frac{(1-\eta) \phi_{j}\left(\mu_{j}, \eta_{j}, \alpha, A, B\right)}{(j-\eta)(A-B)}\right\}^{\frac{1}{j-1}}(j \geq 2)
$$

(3) The function $f(z)$ is convex of $\mu(0 \leq \xi<1)$ in $|z|<r_{3}$ where

$$
r_{3}=\inf _{j}\left\{\frac{(1-\xi) \phi_{j}\left(\mu_{j}, \eta_{j}, \alpha, A, B\right)}{j(j-\xi)(A-B)}\right\}^{\frac{1}{j-1}}(j \geq 2)
$$

Proof. (1) We must show that $\left|f^{\prime}(z)-1\right|<1-\mu$ for $|z|<r_{1}$. We have

$$
\left|f^{\prime}(z)-1\right| \leq \sum_{j=2}^{\infty} j\left|a_{j}\right||z|^{j-1} \text {. }
$$

Thus $\left|f^{\prime}(z)-1\right|<1-\mu$ if

$$
\sum_{j=2}^{\infty} \frac{j}{1-\mu}\left|a_{j}\right||z|^{j-1} \leq 1
$$

By Lemma 2.2, we have

$$
\sum_{j=2}^{\infty} \frac{\phi_{j}\left(\mu_{j}, \eta_{j}, \alpha, A, B\right)}{A-B}\left|a_{j}\right| \leq 1
$$

Hence (3.25) will be true if

$$
\frac{j|z|^{j-1}}{1-\mu} \leq \frac{\phi_{j}\left(\mu_{j}, \eta_{j}, \alpha, A, B\right)}{A-B}
$$

or, if

$$
|z| \leq\left\{\frac{(1-\mu) \phi_{j}\left(\mu_{j}, \eta_{j}, \alpha, A, B\right)}{j(A-B)}\right\}^{\frac{1}{j-1}}(j \geq 2),
$$

which follows from (3.22). Similarly, we can prove (2) and (3). This completes the proof of Theorem 3.6.

\section{Acknowledgements.}

The present investigation was supported by the Natural Science Foundation of Inner Mongolia of China under Grant 2014MS0101. 


\section{References}

[1] S. Shams, S. R. Kulkarni and J. M. Jahangiri, Classes of uniformly starlike and convex functions, Int. J. Math. Math. Sci., 55 (2004), 2959-2961. http://dx.doi.org/10.1155/s0161171204402014

[2] S. Shams and S. R. Kulkarni, On a class of univalent functions defined by Ruscheweyh Derivatives, Kyungpook Math. J., 43 (2003), 579-585.

[3] H. Silverman, Univalent functions with varying arguments, Houston J. Math., 7 (2) (1981), 283-287.

[4] H. M. Srivastava and S. Owa, Certain classes of analytic functions with varying arguments, J. Math. Anal. Appl., 136 (1) (1988), 217-228. http://dx.doi.org/10.1016/0022-247x(88)90127-8

[5] H. Silverman, G. Murugusundaramoorthy and K. Vijaya, A class of functions defined by Dziok-Srivastava operator, Kyungpook Math. J., 49 (1) (2009), 95-106.

[6] S. S. Miller and P. T. Mocanu, Differential Subordinations: Theory and Applications, in: Series on Monographs and Textbooks in Pure and Appl. Math., vol. 255, Marcel Dekker, Inc, New York, 2000.

[7] T. H. MacGreogor, Majorization by univalent functions, Duke Math. J., 34 (1967), 95-102. http://dx.doi.org/10.1215/s0012-7094-67-03411-4

[8] W. Janowski, Some extremal problem for certain famulies of analytic functions, Ann. Polon. Math., 28 (1973), 648-658.

[9] Shu-Hai Li, Huo Tang and Jing-Yu Yang, A new class of analytic functions defined by convolution with varying argument, Tamkang J. Math., 44 (1) (2013), 31-39. http://dx.doi.org/10.5556/j.tkjm.44.2013.944

[10] S. Kanas and A. Wisniowska, Conic regions and $k$-uniform convexity, J. Comput. Appl. Math., 105 (1999), 327-336. http://dx.doi.org/10.1016/s0377-0427(99)000187

[11] S. Kanas and A. Wisniowska, Conic domains and $k$-starlike functions, Rev. Roumaine Math. Pures Appl., 45 (4) (2000), 647-657. 
[12] F. M. Al-Oboudi and K. A. Al-Amoudi, On classes of analytic functions related to conic domains, J. Math. Anal. Appl., 339 (1) (2008), 655-667. http://dx.doi.org/10.1016/j.jmaa.2007.05.087

[13] K. S. Padmanabhan and M. S. Ganesan, Convolutions of certain classes of univalent functions with negative coefficients, Indian J. Pure Appl. Math., 19 (9) (1988), 880889.

[14] A. W. Goodman, On uniformly starlike functions, J. Math. Anal. Appl., 155 (1991), 364-370. http://dx.doi.org/10.1016/0022-247x(91)90006-1

[15] W. C. Ma and D. Minda, Uniformly convex functions, Ann. Polon. Math., 57 (2) (1992), 165-175.

[16] F. Rønning, Uniformly convex functions and a corresponding class of starlike functions, Proc. Amer. Math. Soc., 118 (1) (1993), 189-196. http://dx.doi.org/10.1090/s0002-9939-1993-1128729-7

[17] R. Bharati, R. Parvatham and A. Swaminathan, On subclasses of uniformly convex functions and corresponding class of starlike functions, Tamkang J. Math., 28 (1997), $17-32$.

[18] Wei-Ping Kuang, Yong Sun and Zhi-Gang Wang, On quasi-Hadamard product of certain classes of analytic functions, Bulletin of Mathematical analysis and Applications, 1 (2) (2009), 36-46.

[19] S. S. Eker and S. Owa, Certain classes of analytic functions involving Sălăgean operator, J. Inequal. Pure Appl. Math., 10 (1) (2009), Article 22, 12 pp.

[20] H. M. Srivastava and S. S. Eker, Some applications of a subordination theorem for a class of analytic functions, Applied Mathematics Letters, 21 (4) (2008), 394-399. http://dx.doi.org/10.1016/j.aml.2007.02.032

[21] H. M. Srivastava, S. S. Eker and B. Şeker, A certain convolution approach for subclasses of analytic functions with negative coefficients, Integral Transforms and Special Functions, 20 (9) (2009), 687-699. http://dx.doi.org/10.1080/10652460902749437 
[22] H. M. Srivastava, Shu-Hai Li and Huo Tang, Certain classes of $k$-uniformly close-toconvex functions and other related functions defined by using the Dziok-Srivastava operator, Bulletin of Mathematical Analysis and Applications, 1 (3) (2009), 1-14.

[23] Yong Sun, Wei-Ping Kuang and Zhi-Gang Wang, Properties for uniformly starlike and related functions under the Srivastava-Attiya operator, Applied Mathematics and Computation, 218 (7) (2011), 3615-3623. http://dx.doi.org/10.1016/j.amc.2011.09.002

[24] Shu-Hai Li and Huo Tang, Certain new classes of analytic functions defined by using the Salagean operator, Bulletin of Mathematical Analysis and Applications, 2 (4) (2010), 62-75.

[25] M. K. Aouf, R. M. EL-AShwah, A. A. M. Hassan and A. H. Hassan, On subordination results for certain new classes of analytic functions defined by using the salagean operator, Bulletin of Mathematical Analysis and Applications, 4 (1) (2012), 239246.

[26] R. M. El-Ashwah, M. K. Aouf, A. A. M. Hassan and A. H. Hassan, A new class of analytic functions defined by using salagean operator, International Journal of Analysis, Volume 2013, Article ID 153128, 10 pages. http://dx.doi.org/10.1155/2013/153128

[27] Shu-Hai Li, Huo Tang and En Ao, Majorization properties for certain new classes of analytic functions using the Salagean operator, Journal of Inequalities and Applications, 2013, 2013: 86. http://dx.doi.org/10.1186/1029-242x-2013-86

[28] S. Owa, The quasi-Hadamard products of certain analytic functions, in: H. M. Srivastava, S. Owa (Eds.), Current Topics in Analytic Function Theory, World Scientific Publishing Company, Singapore, New Jersey, London and Hong Kong, 1992, 234-251. http://dx.doi.org/10.1142/9789814355896_0019

[29] A. Schild and H. Silverman, Convolutions of univalent functions with negative coefficients, Ann. Univ. Mariae Curie-Sklodowska Sect. A, 29 (1975), 99-107.

[30] M. K. Aouf, The quasi-Hadamard products of certain subclasses of analytic p-valent functions with negative coefficients, Applied Mathematics and Computation, 187 (1) (2007), 54-61. http://dx.doi.org/10.1016/j.amc.2006.08.102 
[31] J. Dziok, Certain inequalities for classes of analytic functions with varying argument of coefficients, Mathematical Inequalities and Applications, 14 (2) (2011), 389-398. http://dx.doi.org/10.7153/mia-14-31

[32] Z. Nehari, Conformal Mapping, McGraw-Hill Book Company, New York, Toronto and London, 1952.

[33] P. L. Duren, Univalent functions, Springer-Verlag, New York, Berlin, Heidelberg, Tokyo, 1983.

[34] M. S. Robertson, On the theory of univalent functions, Ann. Math., 37 (2) (1936), 374-408. http://dx.doi.org/10.2307/1968451

Received: January 3, 2015; Published: January 25, 2015 